Asociación de Jóvenes Historiadores y Arqueólogos de Murcia

PANTR REL

REVISTA DE CIENCIA

$$
\begin{array}{r}
Y \\
\text { DIDÁCTICA } \\
\text { DE LA HISTORIA } \\
\text { III - } 2^{2} \text { época }
\end{array}
$$




\section{ASOCIACIÓN DE JÓVENES HISTORIADORES Y ARQUEÓLOGOS DE MURCIA}

\section{PANTA REI. REVISTA DE CIENCIA Y DIDÁCTICA \\ DE LA HISTORIA III. $2^{a}$ época}


CONSEJO EDITORIAL: LAURA ARIAS FERRER

ALEJANDRO EGEA VIVANCOS

ANTONINO GONZÁLEZ BLANCO

RAFAEL GONZÁLEZ FERNÁNDEZ

JOAQUÍN LOMBA MAURANDI

ENRIQUE QUINTANA CIFUENTES

GONZALO MATILLA SÉIQUER

JOSÉ ANTONIO MOLINA GÓMEZ

REDACTOR JEFE:

ÁNGEL LUIS GONZÁLEZ TORRES

Depósito legal: MU-966-1995

I.S.S.N. 1136-2464

Edición de Compobell, S.L. Murcia 


\section{ÍNDICE}

AGRADECIMIENTOS

Presentación

Por Alejandro Egea Vivancos, Laura Arias Ferrer

\section{ARTÍCULOS}

Análisis microscópico de la industria lítica: la traceología

Por Ignacio Martín Lerma

Reflexiones en torno al estudio de la economía en Prehistoria

Por Valentín Martínez García

Aplicaciones SIG en el análisis de las sociedades del pasado. Un caso de estudio: Las primeras comunidades campesinas del Levante Peninsular

Por Gabriel García Atiénzar

La situación actual de los estudios de egiptología en España

Por José Javier Martínez García

La vida cotidiana en el Imperio Asirio. Usos y costumbres de un pueblo que conquistó las Cuatro Regiones del Mundo

Por Ángel Luis González Torres

Las formas de intercambio y las estructuras comerciales orientalizantes en la Vega Baja del Segura: dos variables de estudio arqueológico

Por Sara Pernas García 
Evolución de los patrones de asentamiento en época ibérica. Una propuesta de estudio del mundo ibérico murciano a través del análisis del poblamiento

Hispania Tierra de Roma. Organización y gestión del suelo

Por $M^{a}$ Carmen Santapau Pastor

Hacia una arqueología de la España bizantina. Breves notas a propósito del seminario Work in progress

Por Jaime Vizcaíno Sánchez

Reflexiones acerca de un estudio sobre las estancias auxiliares en la arquitectura barroca catedralicia

Por Francisca del Baño Martínez

La investigación sobre fisiognomía y expresión de las pasiones. Objetivos y metodología

Por María del Mar Albero Muñoz 


\title{
ANÁLISIS MICROSCÓPICO DE LA INDUSTRIA LÍTICA: LA TRACEOLOGÍA
}

IGNACIO MARTíN LERMA

\section{Resumen}

El objetivo de este trabajo es divulgar el protocolo habitual de la Traceología, abordando cuestiones metodológicas y algunas aplicaciones realizadas, hasta el momento, en diversos yacimientos arqueológicos.

Palabras clave: Prehistoria, Estudio Funcional, Traceología, Industria Lítica, Arqueología Experimental.

\begin{abstract}
The aim of this work is to communicate and popularize the usual protocol in Traceology, while discussing on methodological issues and on some practical applications which have been essayed in several sites up-to-date.
\end{abstract}

Key words: Prehistory, Functional Studies, Traceology, Lithics, Experimental Archaeology.

\section{Historiografía}

La Traceología es una disciplina analítica, al servicio de la Arqueología, basada en el estudio de las huellas que se generan sobre los instrumentos debido a su utilización. La información obtenida nos aporta un diagnóstico de uso: las zonas activas del útil, el tipo de acción (cortar, raspar, perforar, etc), la materia trabajada (hueso, asta, 
piel, etc) y, más difícilmente, el tiempo estimado. A su vez, un Estudio Funcional, englobaría aspectos más amplios, al tratar de integrar el diagnóstico anterior en una visión con mayor complejidad: la posición del objeto dentro de una cadena operativa y abordar la propia funcionalidad del sitio, profundizando así en los modos de vida de los grupos humanos prehistóricos.

El estudio de la funcionalidad, gracias a su larga trayectoria, está considerado como un elemento totalmente necesario para una mejor comprensión del pasado. Los trabajos de Semenov (1964) y, posteriormente, los de Keeley (1980), sirvieron de base para asentar esta disciplina. Será a partir de los años setenta cuando la observación microscópica de trazas se propague de la mano de investigadores como Anderson-Gerfaud (1981), Mansur (1983, 1986) o Plisson (1985), consolidándose así los principios de la Traceología actual.

Durante los años 70 y 80, la gestación de la Traceología dio lugar a numerosas discusiones metodológicas. Destaca la que se originó en torno a qué medio de observación era el más adecuado: altos versus bajos aumentos (Keeley, 1974; Tringhan et alii, 1974). Este debate no fue trivial teniendo en cuenta que el medio óptico elegido determina el tipo de huella a observar. No obstante, hace tiempo que esta discusión se dejó atrás al ser asumido, por la mayor parte de los traceólogos, que la interpretación más correcta es aquella que se apoya en el recurso a todos los medios para el análisis del mayor número posible de evidencias.

En el transcurso de los estudios traceológicos es inevitable apuntar la importancia de una serie de «tests-ciegos» (blind tests) que sirvieron para contrastar los primeros resultados. El test-ciego consiste en analizar una serie de piezas, talladas y usadas experimentalmente por una tercera persona, sin que el analista tenga conocimiento previo de las condiciones de la experimentación (materias primas, materias trabajadas, acciones empleadas, tiempo, etc). Si bien, no han estado exentos de polémica (Grace et alii, 1988), la mayor parte de los tests-ciegos han dado resultados positivos, confirmando la eficacia del método.

\section{Variables del protocolo}

Todo estudio traceológico requiere un protocolo bien establecido que parte de una fase experimental donde aprendemos el comportamiento de los útiles frente al uso y generamos una colección de huellas de referencia con las que contrastar el material arqueológico. En esta fase es preciso el control de los elementos que intervienen en la experimentación: las variables independientes y las huellas que generan (variables dependientes). El segundo paso es la aplicación de estos conocimientos al material arqueológico. 


\section{Variables independientes}

Son aquellos factores que interaccionan entre sí durante el uso, y son básicamente la materia prima, la materia trabajada, el gesto y el tiempo. La forma en la que cada uno de estos elementos interviene, determina el tipo de huellas resultantes.

Las materias primas condicionan la cantidad y calidad de trazas que podemos encontrar en un útil lítico. Con respecto a las materias trabajadas, la respuesta al uso viene condicionada por los factores intrínsecos de cada materia. La piel, los vegetales frescos o el hueso, por citar ejemplos diversos, provocan estigmas muy diferentes y susceptibles de ser reconocidos, siempre que su desarrollo sea suficiente. Por otra parte, incluso la misma materia, puede variar su comportamiento atendiendo a aspectos como el grado de humedad, caso de las huellas derivadas del trabajo de la piel fresca, seca o húmeda.

Importante también es la evaluación del gesto, ya que condiciona de manera directa el tipo de huellas a desarrollar. La actividad es la forma en la que el útil ha transformado la materia, la cinemática del trabajo. Todo instrumento sometido a un esfuerzo concreto, desarrolla un tipo específico de trazas, cuya lectura correcta nos permitiría identificar tanto el material como la acción desarrollada.

\section{Variables dependientes}

Englobamos dentro de las variables dependientes, las huellas generadas por la confluencia de las variables independientes citadas anteriormente, que son el pulimento, las estrías, el embotamiento y los desconchados.

El pulimento se presenta como una capa brillante, situada sobre el borde activo, y que, en caso de un desarrollo extremo, es observable a simple vista. Los rasgos diagnósticos de cada tipo de pulimento, según la materia trabajada, se manifiestan según va avanzando el trabajo y varían en función de atributos como la topografía, la trama, la compacidad, el brillo, la textura y la presencia de accidentes característicos, entre otros. Se han ido definiendo así pulimentos propios de los cereales (el conocido como «lustre de cereal», visible a simple vista), la madera, el hueso, el asta, la piel fresca y seca, la carne, etc. Aunque no existen tipos rígidos que puedan adscribirse a cada materia, sí existen tendencias generales que pueden seguirse.

Una segunda categoría de huellas son las estrías, asociadas a la interpretación del gesto. Podrían definirse como surcos, depresiones o adiciones que se producen sobre la superficie de los bordes usados. Algunos caracteres de las estrías pueden indicarnos el tipo de acción a partir de su posición respecto al filo activo: son paralelas a él en acciones longitudinales y perpendiculares en acciones transversales. Por otra 


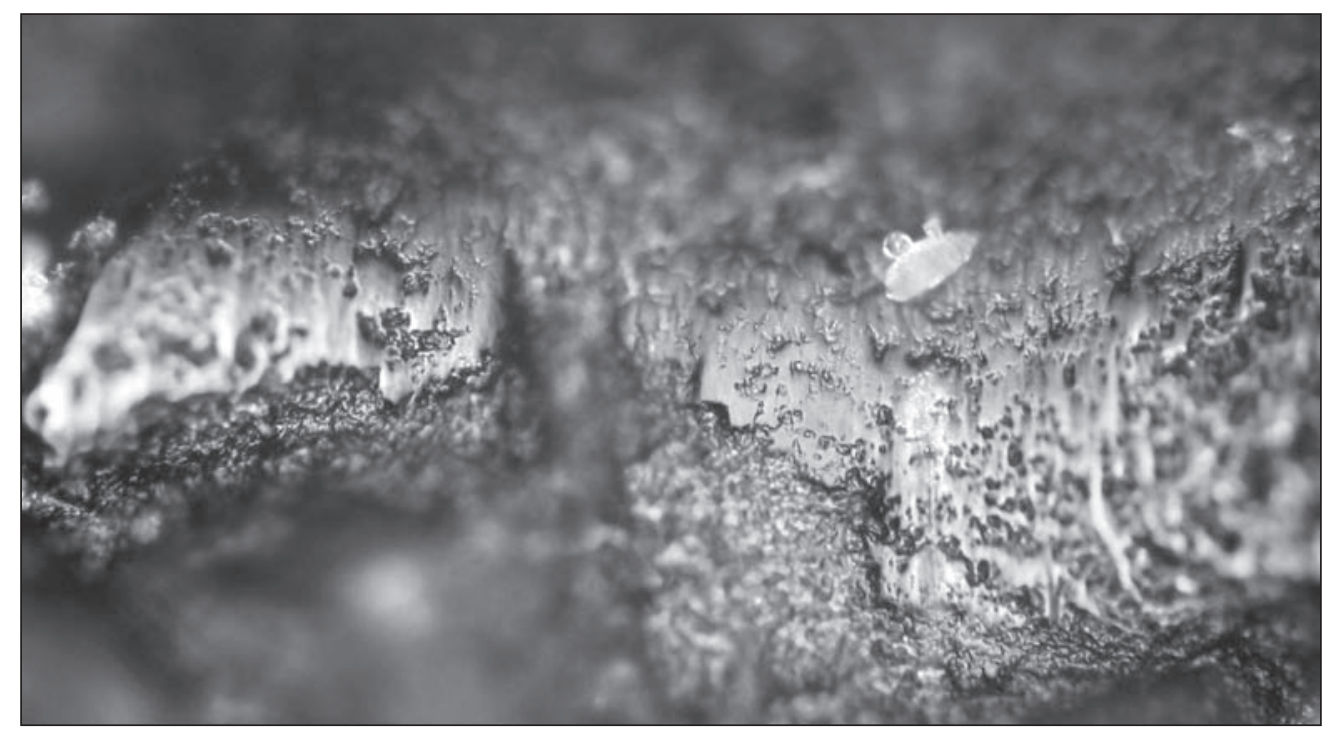

Lámina 1: Pulimento de madera (400x)

parte, determinadas morfologías se asocian a materias trabajadas específicas, si bien de forma no tan estrecha como los pulimentos.

El embotamiento es una variable que no requiere de grandes aumentos para ser reconocida ya que es el redondeamiento que sufren el filo, las aristas y zonas elevadas de la microtopografía silícea, a causa de la abrasión producida durante la acción. Esta huella hay que relacionarla con otros atributos para que la interpretación respecto a la identificación de una materia concreta, sea más precisa.

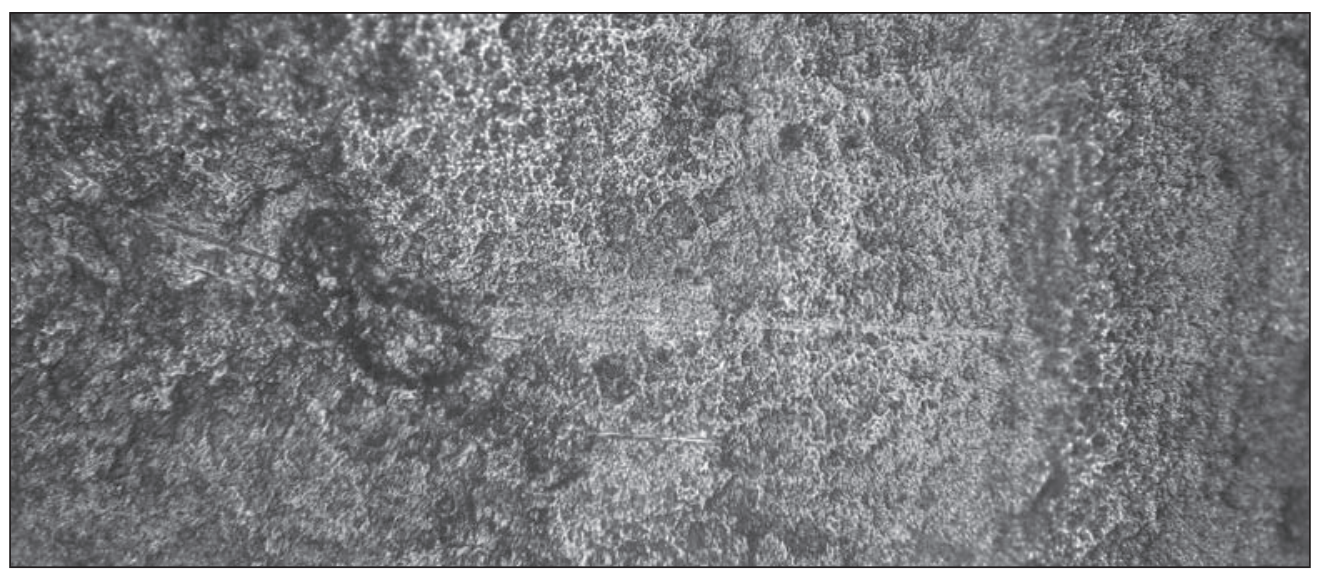

Lámina 2: Estría angosta profunda (200X) 


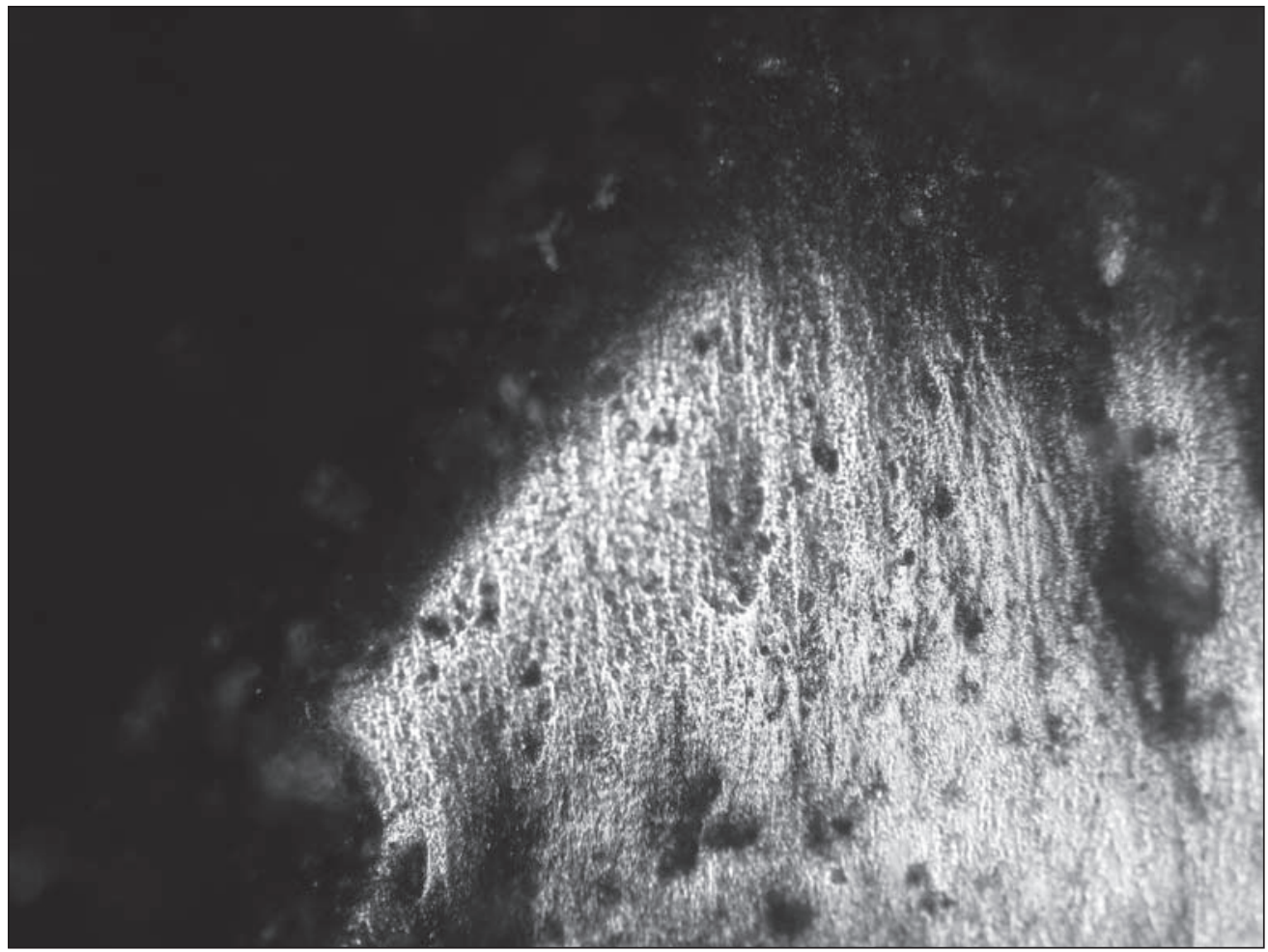

Lámina 3: Embotamiento por trabajo de piel (100X)

Los desconchados son los negativos de las esquirlas o pequeñas melladuras que se van generando durante el trabajo como respuesta a la tensión que sufre la pieza en contacto con la materia trabajada. Pueden ser observados a simple vista y, en todo caso, a bajos aumentos. La dureza de la materia trabajada, los tipos de materias primas y acciones realizadas, la morfología del filo activo, la duración del trabajo y la intensidad de la presión son los factores que van a influir en las características y cantidad de los desconchados, y en estos aspectos se centra su capacidad diagnóstica. Uno de los principales problemas que presentan es su distinción respecto al retoque de talla. Un criterio general para diferenciarlos, sería la regularidad tanto de las extracciones como de su distribución a lo largo de los filos de las piezas, siendo mayor en el caso del retoque intencional.

\section{Metodología}

En los análisis de huellas de uso, se utiliza como medio de limpieza, sobre todo, la cubeta ultrasónica que permite eliminar todo resto de suciedad de las piezas sin que 


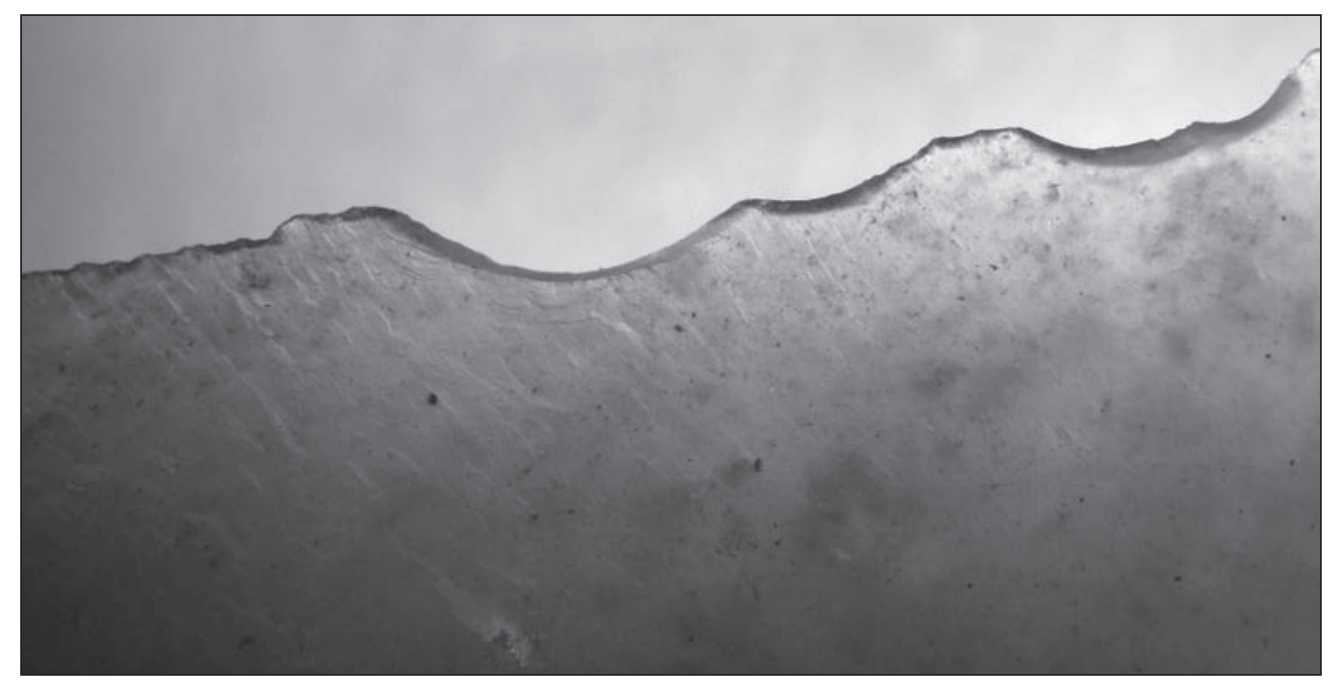

Lámina 4: Desconchados en media luna (4X)

las superficies sufran daños. Para la observación, los aparatos ópticos más comunes son la lupa binocular (que puede llegar a 100x) y se emplea mayoritariamente para el estudio de los desconchados. Por su parte, el pulimento, las estrías y el embotamiento, se analizan con microscópico metalográfico (entre 100x y 800x), que permite un examen más pormenorizado de las superficies silíceas. Por último, el microscópico electrónico de barrido (hasta $8000 \mathrm{x}$ ) suele emplearse para observaciones muy detalladas como, por ejemplo, la detección de residuos.

\section{La experimentación}

La importancia del programa experimental dentro los estudios funcionales radica en que sólo un diseño bien estructurado nos va a permitir conocer la interrelación entre las variables y, en consecuencia, ser utilizado como referencia frente a la colección arqueológica.

Es común en el estudio de los conjuntos líticos prehistóricos, acudir a la experimentación como forma de comprender los aspectos tecnológicos - ¿Cómo fue hecha la pieza?- y los traceológicos - $i$ de que manera fue usada?-. La experimentación permite sentar las bases metodológicas para una correcta interpretación de los restos de la producción lítica y las variantes técnicas que lo originaron (Pigeot, 1991). Se trata de comprender las actividades prehistóricas, en las cuales la elección del instrumento es un factor de primer orden respecto a su adaptación y funcionamiento (Gutiérrez Sáez, 1996). 
Todas las variables del protocolo experimental deben estar perfectamente adecuadas a las condiciones específicas del yacimiento a estudiar, atendiendo a las tres fases de la cadena operativa: selección de la materia prima, proceso tecnológico y funcionalidad del mismo.

Con respecto al primer aspecto, hay que apuntar que, entre la amplia variabilidad de recursos líticos disponibles en una comunidad prehistórica como el sílex, la cuarcita, el cuarzo o la obsidiana entre otras muchas, el empleo del sílex ha sido el más generalizado en los estudios tanto tecnológicos como traceológicos, dado su amplio uso en la Prehistoria y que su composición interna favorece la aparición y el desarrollo de huellas de uso.

Los criterios de reconocimiento en la elaboración de los útiles, que forman parte de una colección experimental, han de identificarse mediante una completa lectura tecnológica (Inizan et alii, 1995) capaz de establecer unos modelos orientativos en las técnicas de desbastado, así como las particularidades que caracterizan el material lítico tallado (composición mineralógica de las materias primas, por ejemplo), las fases de elaboración, su sistema operativo y la valoración de los productos resultantes. Una ayuda inestimable en esta reconstrucción proviene del análisis morfológico y de los remontajes, capaces de identificar la predeterminación y el grado técnico.

El uso, reutilización y abandono del instrumento constituirían la última gran fase de la cadena. Las actividades diseñadas para el desarrollo del programa experimental parten de una serie de modos de actuación que entendemos característicos de los grupos humanos prehistóricos. La amplia variabilidad de objetivos, que implican materias primas, trabajadas y gestos distintos, viene condicionada tanto por el medio natural como por la tradición cultural existente en los que se inserta la comunidad prehistórica analizada, y nos obliga a un diseño específico del protocolo experimental.

La actividad cinegética, por ejemplo, parte de la experimentación de caza, en la que se confecciona el armamento de cazador (puntas de flecha/jabalina/armaduras de diferente morfología insertadas en diversas combinaciones), para, con posterioridad, realizar series de disparos sobre un objetivo y a distancias determinadas. Los resultados se medirán en desperfectos causados en el armamento e índice de penetración, entre otros.

Otros ejemplos de actividades son comunes en los trabajos experimentales realizados hasta el momento. Los procesos de carnicería, con tareas de desollado, descarnado y descuartizado, se han desarrollado con una amplia variedad de animales desde elefantes hasta ciervos y cabras o conejos, entre muchos más.

Las pieles han sido trabajadas en estado seco y fresco, en ocasiones humedecido, con pelo o sin él y con la aplicación de óxidos de hierro o ceniza. El sistema de sujeción de la piel y la tensión ejercida sobre la misma repercute de igual forma en el 


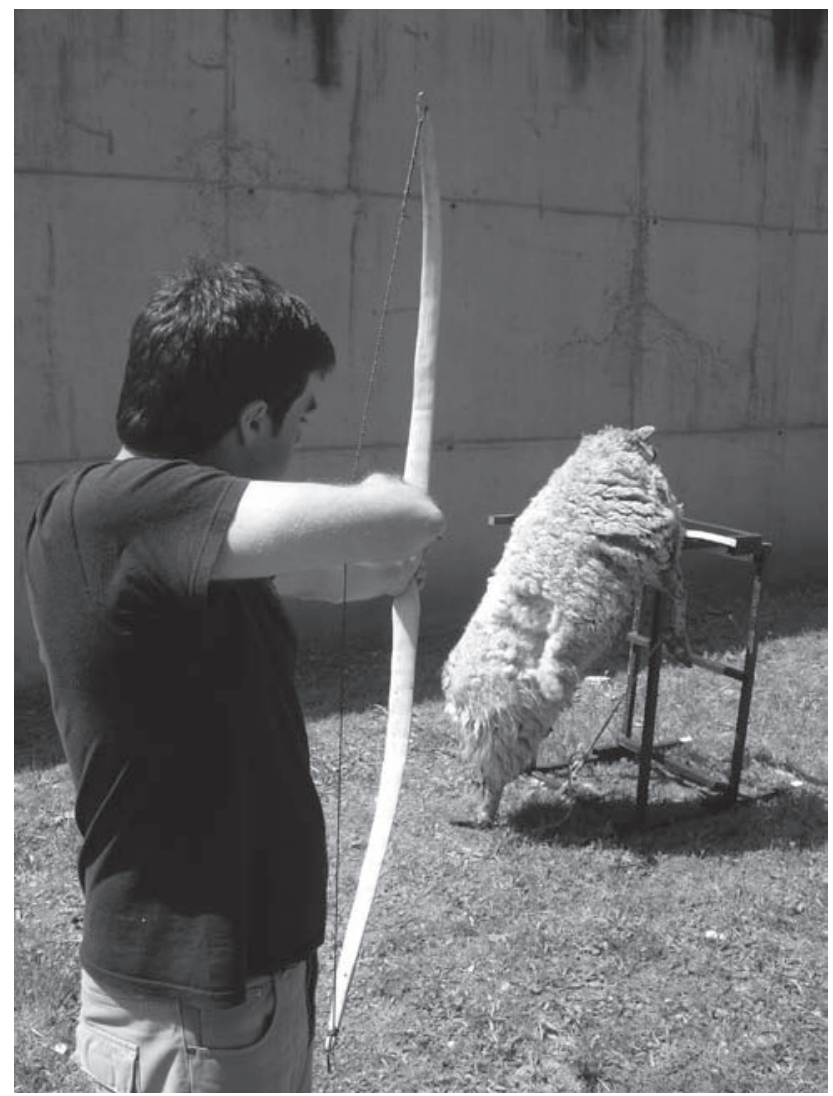

Lámina 5: Experimentación de caza con hojitas de dorso enmangadas lateralmente.

desarrollo y tipo de huellas. El pino, roble o boj, en estado seco o fresco, son algunas de las muchas variedades de maderas empleadas para los estudios traceológicos en Prehistoria.

El hueso, tanto escápulas, tibias como costillas, han sido trabajadas en estado fresco, seco, remojado, humedecido y cocinado. El asta de reno o ciervo es otra de las materias preferentes en las experimentaciones sobre paleolítico, considerando el mayor rendimiento de éstas, remojadas o humedecidas. Los vegetales, los cuales generan la huella conocida como «lustre de cereal», la piedra, los restos malacológicos para objetos de adorno personal o los tendones para cordajes completan la serie de materiales habituales en las experimentaciones.

Todo este desarrollo experimental debe de contar con los útiles adecuados y el conocimiento de los procesos de trabajo, para cuya comprensión, la Etnoarqueología nos aporta una documentación de primer orden. La valoración de los resultados obte- 
nidos, deberá atender no sólo las trazas conservadas en el instrumento sino también los impactos surgidos en la materia transformada.

\section{Conclusiones}

Hasta el momento son numerosos los análisis de huellas de uso que se han aplicado a ámbitos cronológicos y geográficos variados. Estos trabajos han tratado cuestiones muy diversas, con el fin de obtener una mejor comprensión de los asentamientos prehistóricos y arrojar luz a problemas específicos de determinadas etapas (Martín Lerma et alii, e.p.). Las aportaciones de la Traceología a estas problemáticas han sido expuestas en diversas publicaciones (por ejemplo, Anderson et alii, 1987; LeviSala, 1989; Jardón Giner, 1990; Calvo, 2002; González Urquijo e Ibáñez Estévez, 2005/2006; Gibaja, 2007).

Entre otras cuestiones se han abordado las relaciones entre la morfología de los instrumentos y su función, la compresión global de las cadenas operativas (incluyendo temas relativos a la selección y gestión de los recursos líticos, así como la utilización de materias trabajadas, algunas de las cuales, caso de las orgánicas, no dejan evidencias en el registro arqueológico) o la distribución de determinados ítems tipotecnológicos y funcionales (aportando información sobre la delimitación de áreas de actividad, tanto a nivel del micro como del macroespacio).

También, la unión de la experimentación y la documentación etnográfica ha permitido ahondar en procesos de trabajo, poco conocidos hasta el momento (Clemente, 1997).

La búsqueda de información relativa a aspectos sociales, económicos e ideológicos se ha derivado, sobre todo, del análisis de los contextos funerarios. Los elementos fundamentales han sido los propios tipos de objetos, el hecho de que hayan sido usados o no, la identificación de uso y, a partir de aquí, la asociación de estos rasgos a aspectos como el sexo y edad de los individuos o el status social (Gibaja, 2002). En un trabajo reciente, como es el caso del enterramiento múltiple de Cueva Maturras, en Argamasilla de Alba (Ciudad Real), el análisis traceológico ha revelado, entre otras cuestiones, que parte del material lítico había sido usado previamente, y no tallado de manera intencional, para ser depositado como ajuar funerario (Gutiérrez Sáez et alii, e.p.).

Todos estos ejemplos citados anteriormente trascienden el ámbito estricto de la identificación para ayudar a resolver aspectos económicos y sociales específicos de diversos momentos prehistóricos. Por tanto, la Traceología y la Funcionalidad se han convertido en un elemento cada vez más necesario en el proceso de investigación multidisciplinar sobre nuestro pasado. 


\section{Bibliografía}

ANDERSON-GERFAUD, P. Contribution méthodologique à l'analyse des microtraces d'utilisation sur les outils préhistoriques. Thèse $3^{\text {ème }}$ Cycle, $\mathrm{n}^{\circ} 1607$, Université de Bordeaux I, 1981.

ANDERSON-GERFAUD, P; MOSS, E. y PLISSON, H. «A quoi ont-ils servi? L'apport de l'analyse fonctionnelle». B.S.P.F. 84, (8), 1987, 226-237.

CALVO TRÍAS, M. Útiles líticos prehistóricos. Forma, función y uso. Ariel Prehistoria, Barcelona, 2002.

CLEMENTE CONDE, I. Los instrumentos líticos del Túnel VII: una aproximación etnoarqueológica. Treballs d'Etnoarqueología II. CSIC - UAB, 1997.

GIBAJA BAO, J.F. La función de los instrumentos líticos como medio de aproximación socio-económica. Comunidades Neolíticas del V-IV milenio Cal BC en el noreste de la Península Ibérica. Tesis Doctoral. Universitat Autònoma de Barcelona, 2002.

GIBAJA BAO, J.F. «Estudios de Traceología y Funcionalidad». Praxis Archaeologica 2, 2007, 49-74.

GONZÁLEZ URQUIJO, J.E. e IBÁÑEZ ESTÉVEZ, J.J. «El uso del utillaje en piedra en el final del Paleolítico Superior Peninsular». Munibe 57 (2), 2005/2006, 227-238.

GRACE, R.; ATAMAN, K.; FABREGAS, R. y HAGGREN, C.M.B.: «A ultivariate approach to the functional analysis of stone tools». En Beyries, S. (Ed): Industries lithiques. Tracéologie et technologie. BAR internacional Series 411, 1988, 217230.

GUTIÉRREZ SÁEZ, C. Traceología. Pautas de análisis experimental. Temas de Arqueología, 4, FORO, Madrid, 1996.

GUTIÉRREZ SÁEZ, C.; MARTÍN LERMA, I.; MARIN DE ESPINOSA SÁNCHEZ, J.A. y MÁRQUEZ MORA, B. «Industria lítica tallada del ajuar funerario del Abrigo 1 de Cueva Maturras (Argamasilla de Alba, Ciudad Real). Análisis tecnológico y funcional». En Homenaje a D. E. Ripoll Perelló. Espacio, Tiempo y Forma. En prensa.

INIZAN, M.L.; REDURON, M.; ROCHE, H. y TIXIER, J. Technologie de la Pierre taillée. Tome 4. C.N.R.S., 1995.

JARDON GINER, P. «La metodología del análisis traceológico y su aplicación a conjuntos líticos prehistóricos». Saguntum 23, 1990, 9-37.

KEELEY, L.H. «Technique and methodology in microwear studies: a critical review». World Archaeology 5 (3), 1974, 323-326.

KEELEY, L.H. Experimental determination of stone tool use: a microwear analysis. University of Chicago Press. Chicago, 1980. 
LEVI-SALA, I. «Que peuvent vraiment nous révéler les études microscopiques des artéfacts lithiques? ». L'Anthropologie 93 (3), 1989, 643-658.

MANSUR-FRANCHOMME, M.E. Traces d'utilisation et technologie lithique: exemples de la Patagonie. Thèse de 3 cycle présentée à l'Université de Bordeaux, 1983.

MANSUR-FRANCHOMME, M.E. Microscopie du matériel lithique: traces d'utilisation, altérations naturelles, accidentelles et technologiques. Exemples de Patagonie. Cahiers du Quaternaire IX, 1986.

MARTÍN LERMA, I.; MARÍN DE ESPINOSA SÁNCHEZ, J.A. y GUTIÉRREZ SÁEZ, C.: «Estudios funcionales en Prehistoria: ¿Qué información nos aportan los útiles líticos?». Verdolay. En prensa.

PIGEOT, N. «Réflexions sur l'histoire technique de l'homme : de l'évolution cognitive a l'évolution culturelle». Paléo 3, 1991, 167-200.

PLISSON, H. Etude fonctionnelle d'outillages lithiques préhistoriques par l'analyse des micro-usures: recherche méthodologique et archéologique. Thèse de 3eme Cycle, Université de Paris I, Pantheon-Sorbone, Paris, 1985.

SEMENOV, S.A. Prehistoric technology. An experimental study of the oldest tools and artifacs from traces of manufacture and wear. Cory, Adams and Mackay Ltd., Londres, 1964. Traducc. española: Tecnología Prehistórica, 1981, Akal.

TRINGHAM, R.; COOPER, G.; ODELL, G.H.; VOYTEK, B. y WHITMAN, A. «Experimentation in the formation of edge-damage: a new approach to lithic analysis». Journal of Field Archaeology 1, 1974, 171-196. 